\title{
An automated runway: Responding without postreinforcement goal tracking
}

\author{
RONALD J. SEARS \\ Northwestern University, Evanston, Illinois 60201
}

\begin{abstract}
The behaviors of rodents in manual runways are subject to experimenter variability and error and to the stimulus properties of handling. Automated shuttle runways are limited by the shuttle response itself. The response is difficult to learn and maintain because it requires that subjects leave an area associated with reinforcement. This report describes an automated analogue of the traditional straight alley that features unidirectional responding and a goal area physically removed from the runway after each trial. A series of studies demonstrate that rats and gerbils quickly acquire and maintain a stable appetitive running response. This response also provides a sensitive measure for the effects of conditioned fear.
\end{abstract}

The historical and theoretical advantages of the straight alley runway are limited by several methodological problems. In traditional runway studies the experimenter generally handles subjects on every trial, and manually controls the timing of doors, reinforcements, and trial spacing. This provides unwanted stimulus cues. In appetitive training, for example, handling is first paired with placement into the startbox and then it is paired with the subject's removal from the food area. With an escape or avoidance response, analogous problems arise. Small variations in handling often have a major effect on behavior (Candland, Faulds, Thomas, \& Candland, 1960; Levis, 1970; Sperling \& Valle, 1964; Wahlsten \& Sharp, 1969).

In an effort to avoid some of these problems, an automated version of the shuttlebox is often used. With this instrument, the start and goal areas are reversed after each trial, and subjects are required to retrace their previous path to reach the goal on each succeeding trial. It is difficult to train subjects to leave areas where they have received food reinforcement (Frey, Eng, \& Gavin, 1972; Skinner, 1959, p. 365) or approach areas where they have been punished (Bolles, 1970; Theios \& Dunaway, 1964). Also, animals exhibit strong tendencies to approach stimuli associated with appetitive reinforcement and avoid stimuli associated with aversive stimulation even when such responses cost reinforce-

The author wishes to thank William Terris, James J. Vasa, Lawrence E. Lack, and Gordon Berg for their advice in the design and construction of the automated runway. Thanks are due to Peter Frey for his aid in preparing this manuscript and to Roberta Popik, Nadia Wasylyshyn, and Craig Landis, who collected the gerbil data. The gerbil experiment was supported by National Science Foundation Research Grant BMS75-02313. Preparation of this manuscript was facilitated by NSF Research Grant BNS77-00158. Requests for reprints should be directed to the author, 202 Cresap Laboratory, Northwestern University, Evanston, Illinois 60201 . ments (Breland \& Breland, 1966; Hearst \& Jenkins, 1974; Wessells, 1974). Since these competing responses can become stronger with extended training, performance is often poor even after many trials. It is unclear whether subjects are approaching appetitive or avoiding aversive stimuli.

Several solutions have been proposed. One is to place several runways end-to-end in a continuous path so subjects are not required to reverse their direction (Davis, Babbini, \& Huneycutt, 1967; Hanfort, Zimmerman, \& Leckrone, 1967; Stewart \& Anisman, 1970). A second approach (Baum \& Babrow, 1966) is to rotate the entire shuttlebox $180 \mathrm{deg}$ between trials so subjects always run in a single direction away from the same "area." A third approach (Crowell \& Eaton, 1974; Kruger, Galvani, \& Brown, 1969) is to radically alter the former goal area of the shuttlebox when it serves as a startbox on the next shock escape trial. For example, Crowell and Eaton (1974) placed subjects on a trapdoor above a grid floor at one end of the runway; the trial began by dropping the trapdoor and raising a similar platform at the opposite end of the runway. To excape shock, subjects had to run and jump onto the second platform. The process was then repeated in the opposite direction. Subjects could not track the former goal area because it was removed from the runway with each new trial.

The present article describes an automated runway designed for appetitive training of small rodents (rats and gerbils). It resembles a traditional straight alley except that subjects are not handled between trials. The subjects do not have to retrace after each trial and the goal area is removed after the food pellet has been consumed. This instrument permits easy observation and automatic recording of the subjects' responses, is easy to clean, is immune to shorted grids during shock testing, and minimizes odor cues (cf. Wasserman \& Jensen, 1969). 


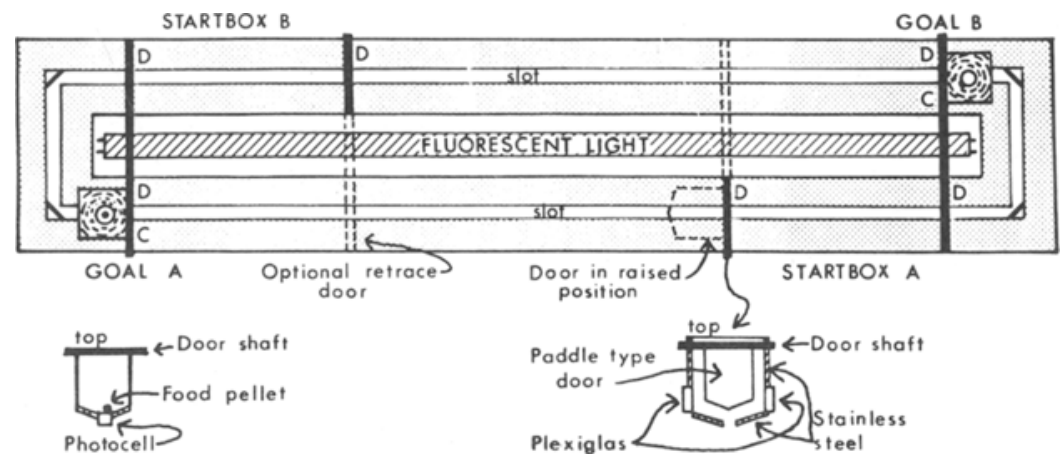

Figure 1. Top view of runway showing locations of various doors (D) and food cups (C). Inserts show cross sections of the alley and a door as well as the food cup.

\section{APPARATUS}

The automated runway (see Figure 1) consists of two parallel runways joined end-to-end, goalbox-to-startbox, such that subjects always make approach responses in the same direction. Access to the various sections of the runways is controlled by electrically activated paddle-type Plexiglas doors which open (i.e., swing through a 90-deg arc up and away from the subject) when the subject is detected in appropriate positions by photocells. The floor of the apparatus consists of two strips of stainless steel $3.2 \mathrm{~cm}$ wide, separated by a $1.6 \mathrm{-cm}$ slot. A metal bar (4 mm diam) is positioned across each of the four corners formed by the outside edge of the floor slot to maintain this slot width at the diagonal gap across the corner. Since the apparatus is narrow ( $7.6 \mathrm{~cm}$ wide) and the floor strips slant $16 \mathrm{deg}$ downward toward the slot in the center, subjects are forced to stand over the slot; therefore, urine and feces rarely remain on the floor and odor cues are minimized. The walls of the apparatus consist of a $2.5 \mathrm{~cm}$ strip of transparent Plexiglas next to the floor, with a $5.1-\mathrm{cm}$ strip of stainless steel above. A 91.4-cm 30-W fluorescent bulb is mounted between the two alleys, adjacent to the inside Plexiglas wall strips. This bulb provides general illumination and acts as a light source for the alley photocells, which are positioned behind the outside Plexiglas wall strips. The top of the runway is covered with transparent Plexiglas for observation and is hinged to allow access.

Each startbox is $30.5 \mathrm{~cm}$ long and contains a waterdrinking tube on the left side of the start door. The goal area of the runway is located $61.0 \mathrm{~cm}$ in front of the startbox door. A recessed food cup (see Figure 2) is mounted in the end wall of the goal area. This end wall also serves as the exit door which allows subjects to move to the startbox of the adjacent alley. A small partition located inside the food cup closes the access port to the food cup as the exit door is raised. Thus, when the door is raised, the goal cup is effectively removed from the runway. The subject can then move to the next startbox without interference from goal-cup tracking responses. The bottom of the food cup is funnel shaped, with a photocell at the center of the funnel. A small bulb (Chicago Miniature No.313) located $10.2 \mathrm{~cm}$ above the photocell provides illumination. This photocell detects the subject's arrival at the goal and activates the pellet dispenser. The photocell also indicates when the subject has removed its head and the pellet from the food cup. A second photocell indicates that the subject is within $1.3 \mathrm{~cm}$ of the end wall. As a precaution against injury, the food-cup-end wall is not raised until both photocells are clear. When the food cup is raised, any remaining food crumbs fall into a small trap. When the cup is next lowered, the crumbs are thrown to the slot in the floor and out of the runway. This prevents the inadvertent triggering of the photocell on the next trial.

The two sides of the stainless steel floor and adjoining walls are electrically isolated from each other and are spaced such that an adult rat cannot stand on one side only. A constant-current ac shock source (series resistance of 2 megohms) is used to administer shock, since Lambert and Hammond (1970) have reported that this shock source yields more reliable performance in a
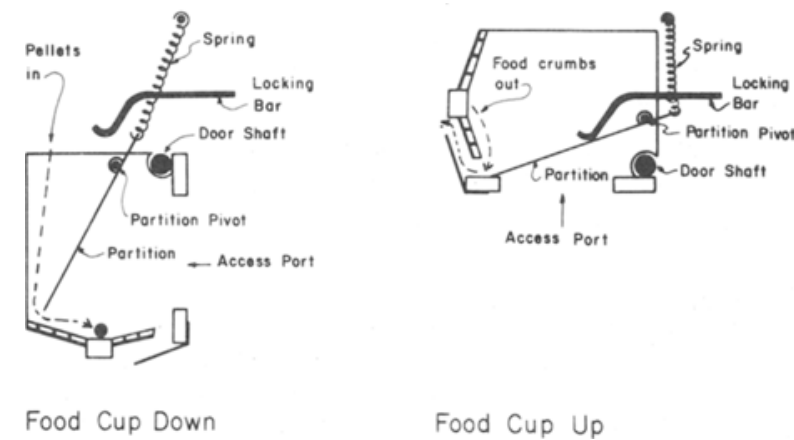

Figure 2. Detailed view of food cup showing placement of the partition with the food cup in the down and up positions. Note the locking bar in the up position which prevents subjects from forcing the partition open. When the food cup is lowered, any food crumbs in the trap are expelled. This same system could also dispose of uneaten pellets if desired. 
slotted-floor apparatus than a matched-impedance shock source. Also, based on Campbell and Masterson (1969), the shock source is set below $500 \mathrm{~V}$ to avoid arcing.

Latency data during any segment of the response are measured automatically by recording the time between interruptions of photocells. Since a fluorescent tube is used, the photocell light source is present along the entire inside wall of the runway, and the photocells can be placed anywhere along the outside Plexiglas wall strip. The continuous light source also avoids discernible photocell light beams that can distract subjects as they pass by. No accuracy is sacrificed by this design, since alley photocells are mounted in small-diameter black tubes and thus are activated only when a sharply defined beam of light across the alley is interrupted.

\section{ACQUISITION AND RESPONSE STABILITY WITH RATS}

Acquisition rate, response stability, and alternative response measures were evaluated with rats. The procedures described are based on a series of pilot studies in which the intertrial interval, amount of reinforcement, and presence or absence of a water bottle were varied within subjects to optimize response speed and stability.

With rats, the presence of a water bottle in each startbox is not arbitrary. Pilot studies indicated that after eating a pellet, rats immediately crossed over to the next startbox and drank when water was available. Without the water bottles, subjects engaged in exaggerated ex ploratory responses and often retraced to the original startbox door after eating a food pellet. This resulted in variations in time between successive trials and partially disrupted performance. Levitsky and Collier (1968) reported that rats showed a preference for drinking water over wheel running immediately after they received a food pellet in a barpressing task. Evidently, the presence of the water bottles in the runway capitalizes on this preference by drawing rats into the next startbox and suppressing adjunctive running behavior.

With a water bottle in the startbox, the duration of the intertrial interval must be at least $15 \mathrm{sec}$. Short intertrial intervals $(5$ to $10 \mathrm{sec}$ ) can result in some rats continuing to drink instead of running for food when the startbox door is opened.

\section{Method}

The subjects were 10 male Holtzman albino rats approximately 71 days old. Subjects were housed individually with free access to water and maintained at $75 \%$ of their original body weight. Two automated runways were enclosed in separate sound-attenuating metal boxes located in the housing room. Constant white noise ( $77 \mathrm{~dB}, \mathrm{SPL})$, humidity (45\%-55\%), and temperature $\left(22^{\circ} \mathrm{C}-25^{\circ} \mathrm{C}\right)$ were maintained in these experimental chambers, as well as the housing room, throughout the experiment. On the first day of the study, subjects were placed in the startbox of a runway; approximately $15 \mathrm{sec}$ later the startbox door opened. When the subject reached the food cup, the startbox door closed and three pellets were dispensed. After the subject had eaten the three food pellets and was clear of the food cup (and a minimum of $2.5 \mathrm{sec}$ had elapsed), the door and food cup assembly opened along with the rear door of the adjacent runway's startbox. When the subject had crossed over and was positioned immediately in front of the adjacent runway's front startbox door, both the rear door of this startbox and the door and food cup assembly of the previous runway closed. Fifteen seconds later, the startbox door opened and the procedure was repeated in the second runway. In this manner, subjects received 20 training trials. On the first few trials of training on Day 1, the alley was baited by hand (three crushed 45 -mg pellets on edge of floor slot) at the middle of each crossover path and at the front of the next startbox. On Day 2, subjects again received 20 training trials and three pellets per trial. On Days 3 through 12, subjects received 40 trials and one pellet per trial. On Day 13, subjects received four trials only.

\section{Results and Discussion}

To demonstrate that subjects quickly and reliably acquired and maintained an asymptotic level of responding, four response measures were examined: start time (leave startbox), approach time (reach goal), goal time (remove food from cup), and crossover time (reach next startbox). These four measures account for all subjectcontrolled time during a trial. Response latencies on each measure were grouped into blocks of 40 trials; the means and standard deviation for Days 1 to 12 are presented in Figure 3. All subjects acquired the response with little variability and reached asymptote on all measures by Day 5 . Start and approach times stabilized by the end of Day 3 . Asymptotic responding was stable across extended training: Analysis of variance across Days 7 to 12 for each measure showed no significant change (all ps $>.10$ ). Figure 3 also presents the mean response times on the four trials of Day 13. Response

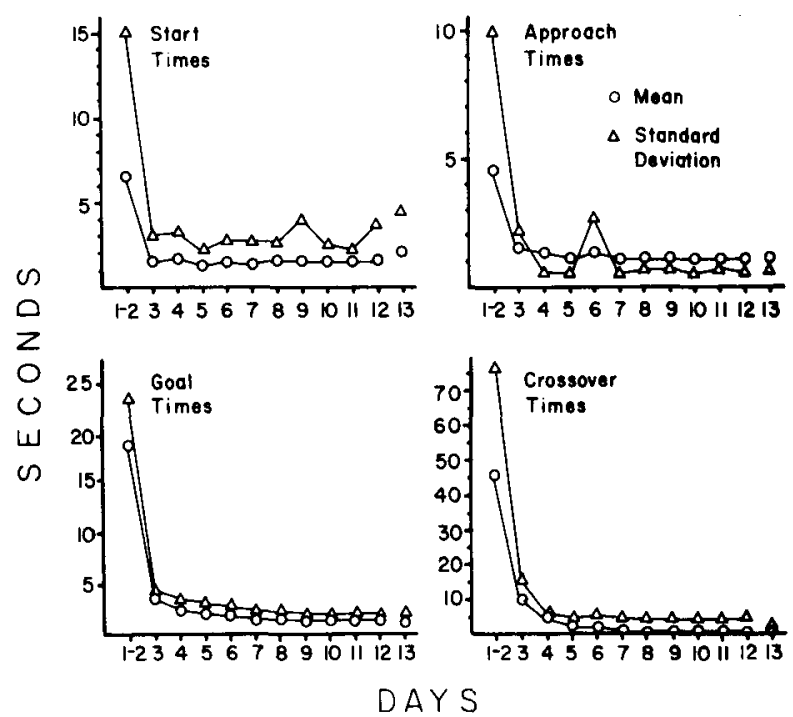

Figure 3. Means and standard deviations of start, approach, goal, and crossover times in blocks of 40 trials (Days 1 to 12) for the 10 rats in the first study. The unconnected points represent the first four trials on Day 13. Note the different ordinate dimensions on each graph. 
measures based on either 6 blocks of 40 trials or 4 trials are quite similar. Each startbox contained a water bottle. While all subjects regularly drank water during the intertrial interval, the total amount consumed in 40 trials was less than $2 \mathrm{ml}$.

\section{ACQUISITION AND CONDITIONED SUPPRESSION WITH GERBILS}

Measurement of the suppression of an appetitive response is often used to evaluate the effectiveness of prior aversive conditioning. One problem with conventional suppression procedures is that the fear conditioned stimulus (CS) occurs in a random relationship with the appetitive response sequence. Since the elicited fear may suppress different segments of the response at different rates, considerable error can occur in single measurements. Therefore, a long-duration CS that extends across many response sequences is often employed. Such CS parameters limit both the range of possible experiments and their interpretation. This study evaluates an alternative procedure using the automated runway. In addition, it provides acquisition data for the gerbil. Since the data are presented in 10 trial blocks rather than 40 , as before, this study also provides a more detailed view of performance during acquisition. Runway acquisition and conditioned suppression following off-the-baseline fear conditioning were evaluated. During suppression testing, a tone previously paired with shock in a separate apparatus was presented just as the startbox door opened. Previous research (Popik, Stern, \& Frey, Note 1, Experiment 2) with this apparatus indicated that a tone explicitly unpaired with a footshock did not disrupt runway performance. So this control group was not included in the present study.

\section{Method}

Subjects. A total of eight male and eight female gerbils ranging from 4 to 12 months in age served as subjects. Subjects were born and raised in a colony maintained at Northwestern University and were individually caged with free access to water throughout the experiment. Subjects were maintained on a 20-h food-deprivation schedule with daily feedings following runway training.

Apparatus. The automated runways were slightly modified. A $3.5-\mathrm{mm}$-diam steel bar was suspended in the center of the slot along the entire runway floor to prevent smaller gerbils from escaping, the water bottles were removed from the startboxes, and an additional speaker was added to each runway enclosure for tone presentations.

For conditioned fear training, subjects were placed in one of four aluminum and Plexiglas boxes $61.0 \mathrm{~cm}$ long, $20.3 \mathrm{~cm}$ high, and $5.1 \mathrm{~cm}$ wide. The boxes had a slotted floor $(1.2 \mathrm{~cm}$ wide) to allow the administration of shock and a speaker for tone presentation. These fear conditioning chambers were located in a large walk-in sound-attenuating chamber in a room separate from the runways.

Procedure. Food deprivation began 5 days before runway training, and, in order to accustom them to the Noyes food pellets, the gerbils were required to eat several each day before receiving their regular food. Acquisition training was similar to that given to rats, except that the intertrial interval was set at

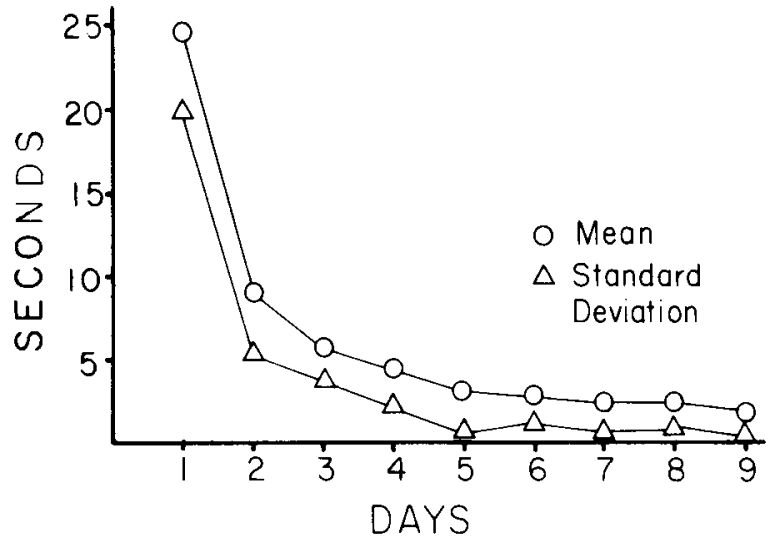

Figure 4. Means and standard deviations of the sum of start and approach times during training in Study 2. The data are based on daily blocks of 10 trials for 16 subjects.

$5 \mathrm{sec}$ and subjects received 10 trials per daily session and only one pellet per trial. Also, the runway was not hand-baited on Day 1 of acquisition, as before. Subjects received 9 days of runway training before testing. On Days 4 to 9 , after daily runway training and $4 \mathrm{~h}$ access to food, subjects received offthe-baseline fear conditioning in the second apparatus. Subjects were placed in the fear conditioning chamber; 12 min later, an 85-dB (SPL) 2,000-Hz tone was presented for $5 \mathrm{sec}$, followed immediately by a $4-\mathrm{mA}$ footshock of .5 -sec duration. Subjects received a single fear conditioning trial each day for 6 days. Suppression testing was conducted on Days 10 and 11. During testing, subjects received 10 runway trials, as before, except that on Trials 4 and 7, the tone was presented for $5 \mathrm{sec}$ beginning when the startbox door opened.

The sum of start and approach times was used to evaluate subjects' performance. Since the conditioned fear elicited by the tone would be expected to disrupt both start and approach times, a composite of these response measures was appropriate.

\section{Results and Discussion}

The gerbils' performance during the 9 days of training is presented in Figure 4. All subjects quickly acquired the response with little variability. Subjects showed an orderly decrease in response time over the first 50 trials, as represented by mean performance on daily blocks of 10 trials. A comparison of the gerbils' overall mean response time on the last 5 days of acquisition $(2.54 \mathrm{sec})$ with similar data (sum of start and approach times) from the last 5 days of training in the rat study $(2.59 \mathrm{sec})$ indicates that gerbils respond similarly to rats in the runway. Since subjects' mean response times on the last 5 days of training show a slight decrease from 3.0 to $2.0 \mathrm{sec}$, it is evident that off-the-baseline fear conditioning had no serious effects on runway responses. Figure 5 presents subjects' mean trial-by-trial performance during CER testing on Day 10. The tone stimulus reliably disrupted responding when presented on Trials 4 and 7, while it had no serious carry-over effects on normal trials. Similar results were obtained when testing was repeated on Day 11.

\section{GENERAL DISCUSSION}

The results of these two studies clearly demonstrate the 


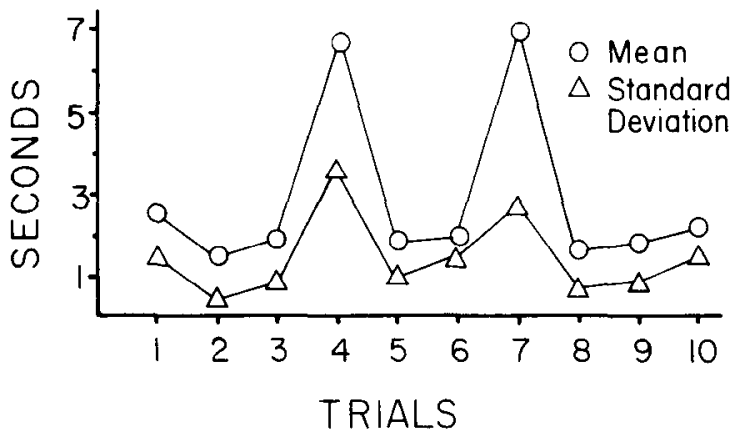

Figure 5. Means and standard deviations of the sum of start and approach times during individual trials on Suppression Test Day 10. The tone was presented as the startbox door opened on Trials 4 and 7 . Each data point represents 16 gerbils.

potential usefulness of the automated runway. Acquisition is rapid with low subject variability, and asymptotic performance is stable with both rats and gerbils. The response established is also robust, as indicated by subjects' immediate recovery from partial response suppression. The suppression test shows low subject variability and can be expected to provide sensitive measurements. The instrument is also very time efficient, allowing the experimenter to reliably administer 40 runway trials to rats in less than $17 \mathrm{~min}$ and 10 runway trials to gerbils in less than $5 \mathrm{~min}$.

To date, over 250 animals have been trained with the runway. In one experiment with rats (Sears, 1976), transfer effects from various drug treatments during asymptotic responding were demonstrated during undrugged tests for response persistence. Both extinction and punishment testing showed low subject variability. In a second study with gerbils and off-the-baseline fear conditioning (Popik, Stern, \& Frey, Note 1, Experiment 2), the instrument proved sensitive to the manipulation of interstimulus intervals on second-order fear conditioning trials.

Experience with the runway has suggested several potential improvements in design. First, the tendency for subjects to retrace can be eliminated by installing a retrace door in the goal area of the runway. The door can be at tached easily to the same shaft as the startbox door of the opposite runway. Both of these doors can then be lowered when subjects reach the goal cup (see Figure 1). Second, acquisition rates can probably be improved with the use of silent mechanisms (perhaps small air cylinders) to activate the doors. Electric motors and solenoids whine and click, and these noises tend to startle some subjects on the first day of training. Finally, the number of acquisition days and trials per day for rats can probably be reduced to 4 days at 20 trials per day with little consequence to response stability.

The instrument described here is very similar to the traditional straight alley and offers the control and efficiency of automation, while avoiding problems associated with shuttle alleys. Use of the automated runway will facilitate generalization from earlier straight-alley studies, while allowing more accurate measurement and a considerable savings in man hours.

\section{REFERENCE NOTE}

1. Popik, R., Stern, S., \& Frey, P. W. Second-order conditioning: The interstimulus interval and the procedure for intermixing first-order and second-order trials, unpublished manuscript.

\section{REFERENCES}

Baum, M., \& Babrow, S. A. An automated analogue of the one-way Miller-type avoidance box. Psychonomic Science, $1966,5,361-362$.
Bolles, R. B. Species specific defense reactions and avoidance learning. Psychological Review, 1970, 77, 32-48.

Breland, K., \& Breland, M. Animal behavior. New York: Macmillan, 1966.

Campbell, B. A., \& Masterson, R. A. Psychophysics of punishment. In B. A. Campbell \& R. M. Church (Eds.), Punishment and aversive behavior. New York: AppletonCentury-Crofts, 1969. Pp. 3-42.

Candland, D. K., Faulds, B., Thomas, D. B., \& Candland, $M$. $H$. The reinforcing value of gentling. Journal of Comparative and Physiological Psychology, $1960,53,55-58$.

Crowell, C. R., \& Eaton, N. K. A fully automated two-way locomotor training apparatus. Behavior Research Methods \& Instrumentation, 1974, 6, 19-22.

Davis, W. M., Babbini, M., \& Huneycutt, B. D. A new apparatus for one-way locomotor avoidance without handling. Psychonomic Science, 1967, 8, 185-186.

Frey, P., Eng, S., \& Gavin, W. Conditioned suppression in the gerbil. Behavior Research Methods \& Instrumentation, $1972,4,245-249$.

Hanford, P. V., Zimmerman, J., \& Leckrone, W. R. An automated runway and Skinner box apparatus. Psychonomic Science, 1967, 8, 99-100.

HeARst, E., \& Jenkins, H. M. Sign-tracking: The stimulusreinforcer relation and directed action. Austin. Tex: Psychonomic Society, 1974.

Kruger, B. M., Galvani, P. F., \& Brown, J. S. A comparison of simulated one-way and shuttle avoidance in an automated apparatus. Behavior Research Methods \& Instrumentation, 1969, 1, 143-147.

LAMBERT, J. V., \& HAMmond, L. J. Effects of shock source and method of contact upon runway escape behavior. Behavior Research Methods \& Instrumentation, 1970, 2, 11.13.

LEVIs, D. J. Between-trial transporting of animals: A methodological consideration. Behavior Research Methods \& Instrumentation, 1970, 2, 157-160.

Levitsky, D., \& Collier, G. Schedule-induced wheel running. Physiology and Behavior, 1968, 3, 571-573.

SEARS, R. J. Resistance to punishment and extinction following responding under methamphetamine or secobarbital. (Doctoral dissertation, DePaul University, 1976). Dissertation Abstracts International, 1976, 37, 1948-B. (University Microfilms No. 76-23,713)

SkInNer, B. F. A case history of scientific method. In S. Koch (Ed.), Psychology: A study of a science (Vol. 2). New York: McGraw-Hill, 1959.

Sperling, S. E., \& Valle, F. P. Handling-gentling as a positive secondary reinforcer. Joumal of Experimental Psychology, 1964, 67, 573-576.

Stewart, D. J., \& Anisman, H. An automated one-way avoidance apparatus. Perceptual and Motor Skills, 1970, 31, 545-546.

Theios, J., \& Dunaway, J. E. One-way vs. shuttle avoidance conditioning. Psychonomic Science, 1964, 1, 251-252.

Waflsten, D., \& SHARP, D. Improvement of shuttle avoidance by handling during the intertrial interval. Journal of Comparative and Physiological Psychology, 1969, 67, 252.259.

Wasserman, E. A., \& Jensen, D. D. Olfactory stimuli and the "pseudo-extinction" effect. Science, 1969, 166, 1307-1309.

Wessells, M. G. The effects of reinforcement upon the prepecking behaviors of pigeons in the autoshaping experiment. Journal of the Experimental Analysis of Behavior, 1974, 21, 125-144. 